\title{
Post-Stenotic Recirculating Flow May Cause Hemodynamic Perforator Infarction
}

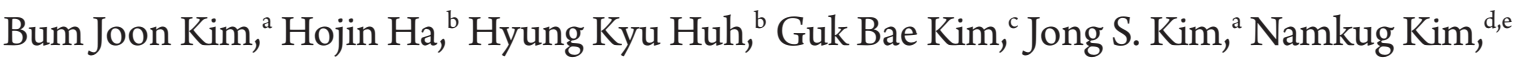 \\ Sang-Joon Lee, ${ }^{\mathrm{b}}$ Dong-Wha Kang, ${ }^{\mathrm{a}}$ Sun U. Kwon ${ }^{\mathrm{a}}$ \\ ${ }^{a}$ Department of Neurology, Asan Medical Center, University of Ulsan College of Medicine, Seoul, Korea \\ ${ }^{b}$ Center for Biofluid and Biomimic Research, Department of Mechanical Engineering, Pohang University of Science and Technology, Pohang, Korea \\ 'Asan Institute of Life Science, Asan Medical Center, Seoul, Korea \\ ${ }^{\mathrm{d}}$ Department of Radiology, Asan Medical Center, University of Ulsan College of Medicine, Seoul, Korea \\ eDepartment of Convergence Medicine, Asan Medical Center, University of Ulsan College of Medicine, Seoul, Korea
}

Background and Purpose The primary mechanism underlying paramedian pontine infarction (PPI) is atheroma obliterating the perforators. Here, we encountered a patient with PPI in the post-stenotic area of basilar artery (BA) without a plaque, shown by high-resolution magnetic resonance imaging (HR-MRI). We performed an experiment using a 3D-printed BA model and a particle image velocimetry (PIV) to explore the hemodynamic property of the post-stenotic area and the mechanism of PPI.

Methods 3D-model of a BA stenosis was reconstructed with silicone compound using a 3Dprinter based on the source image of HR-MRI. Working fluid seeded with fluorescence particles was used and the velocity of those particles was measured horizontally and vertically. Furthermore, microtubules were inserted into the posterior aspect of the model to measure the flow rates of perforators (pre-and post-stenotic areas). The flow rates were compared between the microtubules.

Results A recirculating flow was observed from the post-stenotic area in both directions forming a spiral shape. The velocity of the flow in these regions of recirculation was about one-tenth that of the flow in other regions. The location of recirculating flow well corresponded with the area with low-signal intensity at the time-of-flight magnetic resonance angiography and the location of PPI. Finally, the flow rate through the microtubule inserted into the post-stenotic area was significantly decreased comparing to others $(P<0.001)$.

Conclusions Perforator infarction may be caused by a hemodynamic mechanism altered by stenosis that induces a recirculation flow. 3D-printed modeling and PIV are helpful understanding the hemodynamics of intracranial stenosis.
Correspondence: Sun U. Kwon Stroke Center and Department of Neurology, Asan Medical Center, University of Ulsan College of Medicine, 88 Olympic-ro 43-gil, Songpa-gu, Seoul 05505, Korea

Tel: $+82-2-3010-3960$

Fax: +82-2-474-4691

E-mail: sukwon@amc.seoul.kr

Received: August 30, 2015

Revised: October 5, 2015

Accepted: October 17, 2015

This work was supported by the Korea Health Technology R\&D Project, Ministry for Health \& Welfare, Republic of Korea (HI12C1847), the National Research Foundation of Korea under a grant funded by the Korean government (MSIP) (No. 2008-0061991) and National Research Foundation of Korea (NRF) funded by the Ministry of Science, ICT \& Future Planning (NRF-2015R1A2A2A04003034).

The authors have no financial conflicts of interest.

Keywords Cerebral blood flow; Hemodynamics; Perforator infarcts; Experimental

\section{Introduction}

The pathomechanisms of pontine infarction are diverse, as shown by the lesion patterns and the patency of the involved arteries. ${ }^{1}$ Paramedian pontine infarction (PPI), which extends to the basal surface of the pons, is often associated with the occlusion of perforating arteries by atherosclerotic plaques in the basilar artery (BA), which can be detected by high-resolution magnetic resonance imaging (HR-MRI). ${ }^{2}$ Usually, these BA plaques obliterate the perforator at the most stenotic portion. ${ }^{3}$ 
However, perforator infarctions which occur at the area inconsistent to the area with parental artery stenosis are noticeable and the recirculating flow in the post-stenotic area was conceptually thought to influence the occurrence of perforator infarction. ${ }^{4}$ We recently encountered a patient without a plaque in the region of the PPI, but with a severe stenosis of the BA immediately proximal to the PPI, as confirmed by HR-MRI. The mechanism of PPI in this patient was assessed experimentally using a 3D-printed BA stenosis model and particle image velocimetry (PIV).

\section{Methods}

\section{Case}

An 81-year-old hypertensive and hyperlipidemic woman visited the emergency department after developing dysarthria and subtle right-sided weakness 2 days earlier. She had also experienced transient dysarthria 9 days prior to this visit. Diffusion-weighted imaging showed an acute infarction in the left paramedian area, near the junction of the midbrain and the pons (Figure 1A). Time-of-flight magnetic resonance angiography (TOF-MRA) revealed a severe stenosis at the midbasilar trunk (Figure 1B).

HR-MRI was performed to determine the exact level of the PPI and its relationship to the BA stenosis. The proton-density image showed perforators supplying the infarcted area branching from the upper pons level of the BA (Figure 1C; arrowheads), with the infarcted area extending to the base of the pons at the particular level. However, the atherosclerotic plaque was located in the narrowed BA at the mid-pontine level, but did not obliterate any perforators causing the PPI (Figure 1D). Although the diameter was restored after the area of stenosis, TOF-MRA showed a low signal intensity area at the inner curvature of the post-stenotic area, where the perforator - which supplies the area of the PPI - branches (lines C and D; Figure 1B). The patient underwent a conventional angiogram (Figure $1 \mathrm{E})$ and the result of angiogram was reconstructed in 3 dimensional images (Figure 1F). An experiment with 3D-vascular model was performed to verify the mechanism of stroke. We have used the source image of MRI, which is non-invasive and widely used to evaluate intracranial atherosclerosis to reconstruct the 3D-model, for broad application of this method. The Institutional Review Board of our center approved the study, and a written informed consent was obtained from the patient

\section{Preparing a 3D-model of BA stenosis}

A 3D-model of BA stenosis was reconstructed by a in-house software from the source image of HR-MRI (Figure 2A). ${ }^{5}$
Unnecessary parts of the model were removed, and the surface was smoothed automatically. In detail, the average value of the background intensity outside of BA was initially used as an initial threshold to extract BA from the source image. The threshold value was refined within $\pm 10 \%$ to optimize the separation between the outer tissue region and BA. After constructing the 3D surface model of BA, unnecessary parts of the model were removed by using sculpting function. Finally the surface was smoothed with Laplacian smoothing filter (MeshLab, v1.1). The region of interest was selected from the distal vertebral artery to the distal part of the BA, including the location of the PPI. The reconstructed 3D-model of BA stenosis was fabricated with acrylonitrile butadiene styrene thermoplastic using a 3D-printer (Fortus 400mc, Stratasys; Figure $2 \mathrm{~B}$ ), and was cast using a polydimethylsiloxane silicone compound. The acrylonitrile butadiene styrene model was dissolved out from the polydimethylsiloxane by placing the model in acetone (Figure 2C).

The fabricated BA model was magnified by 5 times in dimension than the actual physiologic site to make fluid-dynamic experiments more convenient. The effect of the change in scale on fluid-dynamic characteristics could be compensated by adjusting other fluid-dynamic parameters, such as flow velocity and viscosity, based on the non-dimensional Reynolds number $(R e)$.

Transcranial Doppler ultrasonography and MRI of the patient showed that the mean velocity of the BA was $13 \mathrm{~cm} / \mathrm{sec}$ and its mean diameter was $3 \mathrm{~mm}$, corresponding to $R e=130$ based on the kinematic viscosity of $3.0 \times 10^{-6} \mathrm{~m}^{2} / \mathrm{s}$. The fluiddynamic experiment utilized working fluid composed of $60 \%$ pure glycerol and $40 \%$ deionized water $(\mathrm{w} / \mathrm{w})$, with a refractive index of 1.413 , resulting in a kinematic viscosity of $8.7 \times$ $10^{-6} \mathrm{~m}^{2} / \mathrm{s}$. Therefore, the average velocity in the polydimethylsiloxane model was adjusted to $7.54 \mathrm{~cm} / \mathrm{s}$ for $R e=130$.

An acryl reservoir was filled with $4 \mathrm{~L}$ of working fluid. A 15 W centrifugal pump, controlled by a mechanical fluid valve, was used to circulate the working fluid at a constant flow rate. The working fluid was seeded with poly-methylmethacrylate fluorescence particles (poly-methylmethacrylate-Rhodamine B-Particles, Dantec Dynamics, Denmark) with a diameter of 20-50 $\mu \mathrm{m}$. All experiments were performed at a controlled room temperature of $25^{\circ} \mathrm{C}$.

\section{PIV velocity field measurements}

A $0.5 \mathrm{~mm}$-thick laser sheet was generated using a $1 \mathrm{~W}$ continuous diode-pumped solid state laser (Shanghai Dream Lasers Technology Co., Ltd., China) to illuminate the measurement plane (Figure 2D). Two high-speed cameras (Fastcam 

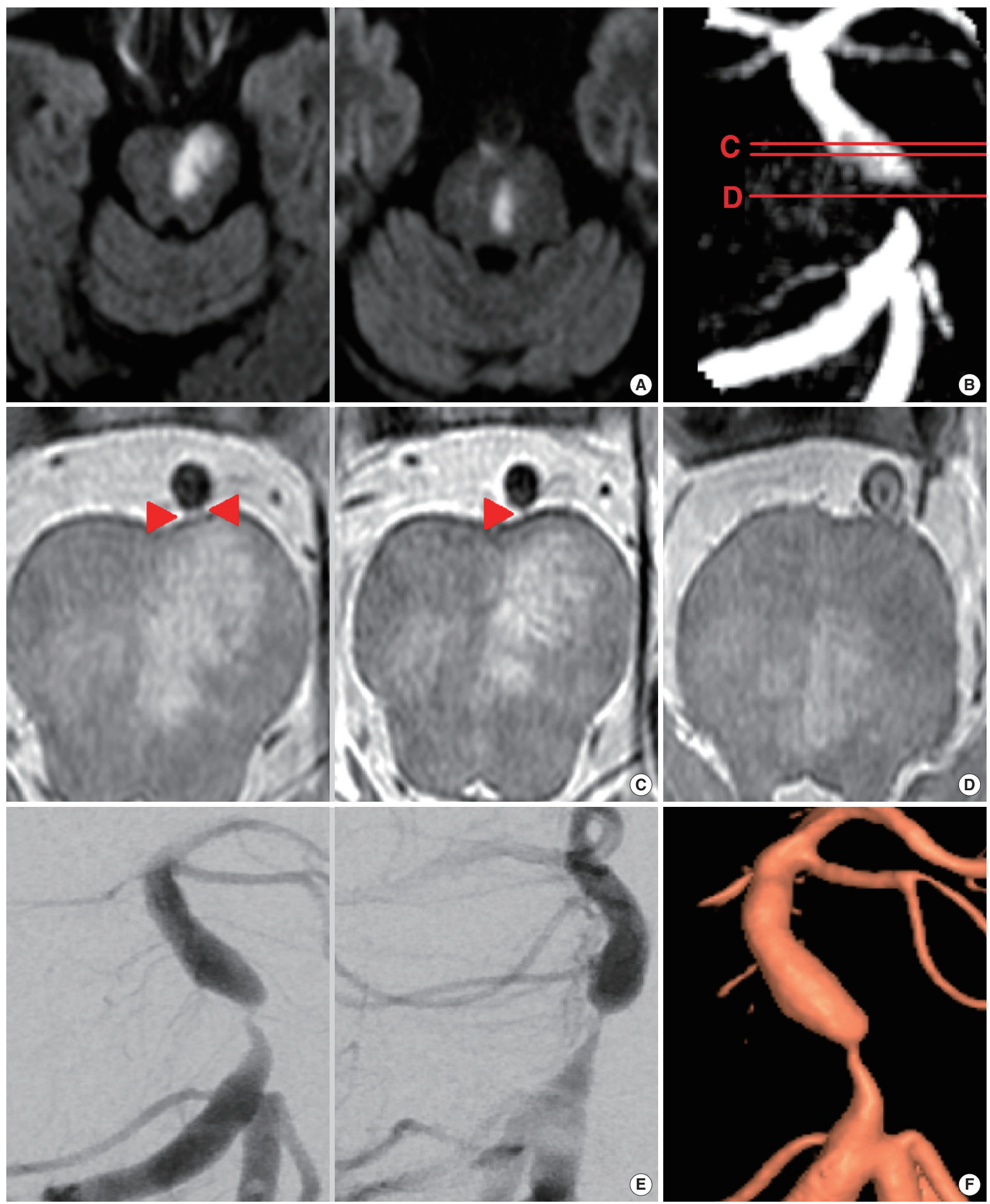

Figure 1. Imaging results of the patient with paramedian pontine infarction (PPI). (A) Diffusion weighted image, (B) time-of-flight magnetic resonance angiography (TOF-MRA) with lines indicating the levels of high-resolution magnetic resonance imaging (HR-MRI), (C) HR-MRI at the levels of the PPI with perforators indicated by arrowheads, (D) most severe level of basilar artery (BA) stenosis, and (E) Conventional angiogram with (F) 3D-reconsturction. 

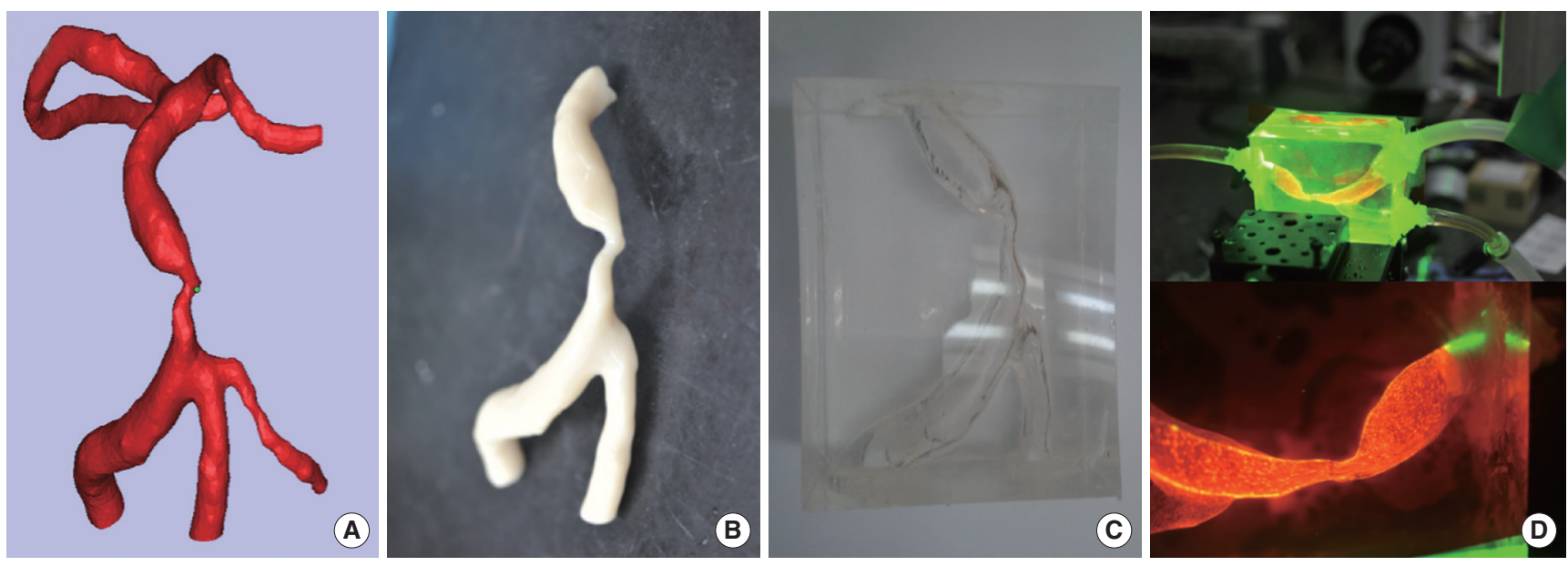

Figure 2. 3D-printed vascular stenosis model and particle image velocimetry (PIV). (A) 3D-reconstructed basilar artery (BA), (B) 3D-printed fabricated model, (C) the silicon cast, and (D) blood flow measurements using PIV.

SA1.1, Photron, USA) at different positions were used to measure velocity fields in the horizontal and vertical planes. The PIV data were acquired from 55 planes at gaps of $0.5 \mathrm{~mm}$ from both directions. A high-speed camera captured 352 pairs of flow images at 3,000 frames per second. A total of 351 instantaneous vector fields were obtained in each measurement plane, and were statistically averaged to determine the mean velocity fields.

PIV analysis was performed using PIVview (PIVview 2C, PIVTEC, Germany). Instantaneous velocity fields were determined by applying a fast Fourier transform-based cross-correlation PIV algorithm to the flow images. A multi-grid interrogation window scheme was adopted with $50 \%$ overlapping. Additional details about the principles and uncertainties of this PIV system have been described. ${ }^{6}$

\section{Flow rate measurement through microvessels}

Considering the diameter of the pontine perforators, microvessels $3 \mathrm{~mm}$ in diameter were inserted into the posterior aspect of the BA stenosis model at three points; pre-stenosis and post-stenosis with and without recirculating areas (Figure 3B). The flow rates through these three microvessels were estimated by measuring the amount of fluid transport through each during a set time period. Flow rates were measured five times, and the results were analyzed statistically using a $t$ test (Minitab 15, Minitab Inc., PA, USA). The level of significance was defined as $P<0.01$.

\section{Results}

PIV measurements of the flow field through the horizontal and vertical planes showed a recirculating flow immediately distal to the stenotic portion (Figure $3 \mathrm{~A} ; \mathrm{H}=17$ ). The velocity of the flow in these regions of recirculation was slow as onetenth that of the flow in other regions. Due to the non-planar configuration of the BA stenosis model, blood flow at the poststenotic region has a spiral shape, allowing the recirculating motion of the flow to be observed in both planes - horizontal and vertical (Figure 3A). Measurement of flow rates through microvessels showed that the rate was significantly lower in the post-stenotic area with recirculation than in the pre-stenotic area or the post-stenotic area without recirculation $(P<0.001$; Table 1, Figure 3B and C).

\section{Discussion}

This study describes a patient with a PPI at the post-stenotic area of the BA, but without a plaque obliterating the orifice of the BA perforators, as shown by HR-MRI. The hemodynamic status of the intracranial artery and the mechanism of stroke were verified experimentally using a 3D-printed BA model and PIV. Finally, this 3D-printed model showed a recirculating flow in the post-stenotic area decreasing the flow through the microtubule inserted in the particular area posteriorly to the BA.

Alterations in post-stenotic hemodynamics may have contributed to occurrence of PPI in the patient described here. As the blood vessel diameter increases in post-stenotic areas, flow velocity decreases and pressure increases, resulting in blood flowing against a pressure gradient. Finally, the outer layer of blood flow detaches from the main area of blood flow, forming a recirculating zone. ${ }^{78}$ In this area, the relative low velocity recirculating flow is characterized with high vorticity (local rotational fluid motion) in the core region, which causes locally a low pressure field around the recirculating flow. ${ }^{9,10}$ Conse- 

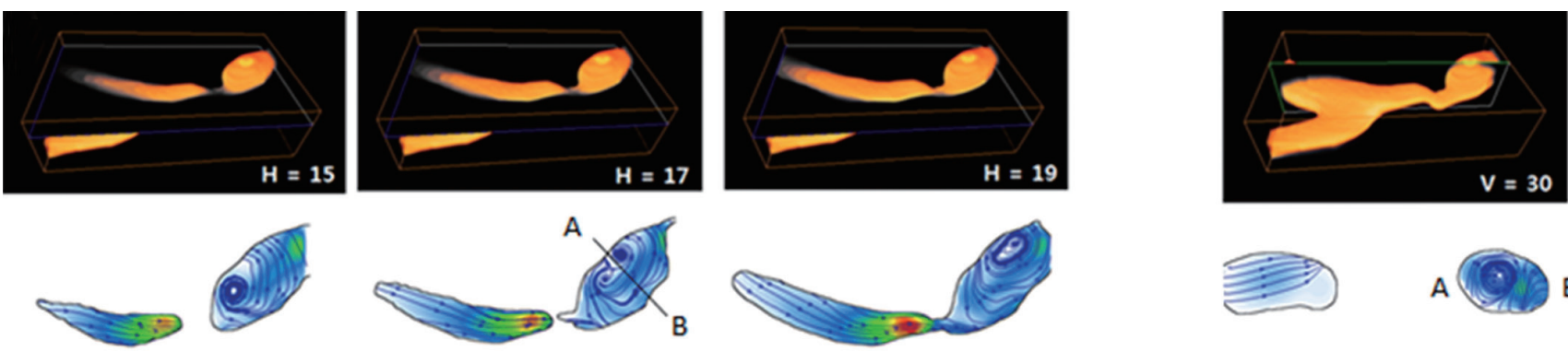

A

\section{[Horizontal plane]}

[Vertical plane]

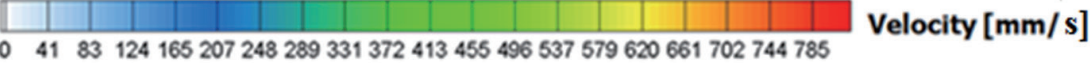
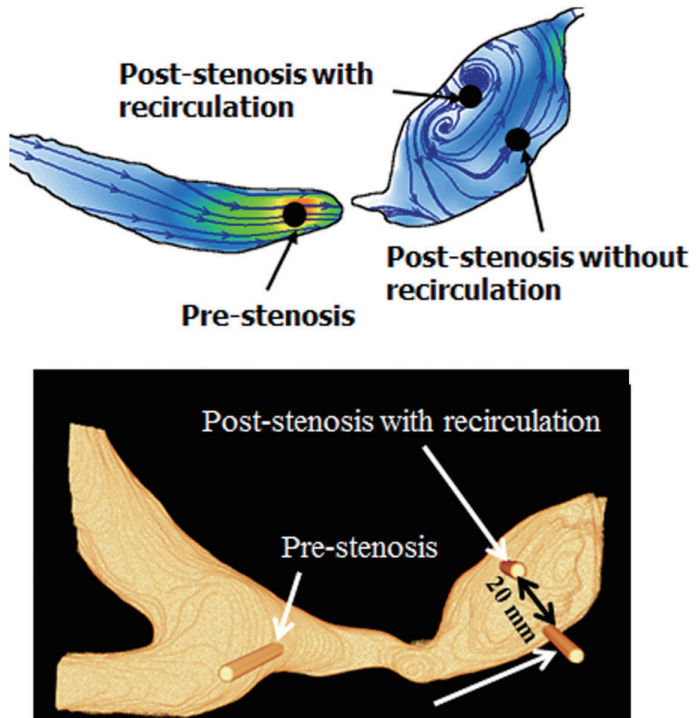

Post-stenosis without recirculation
B

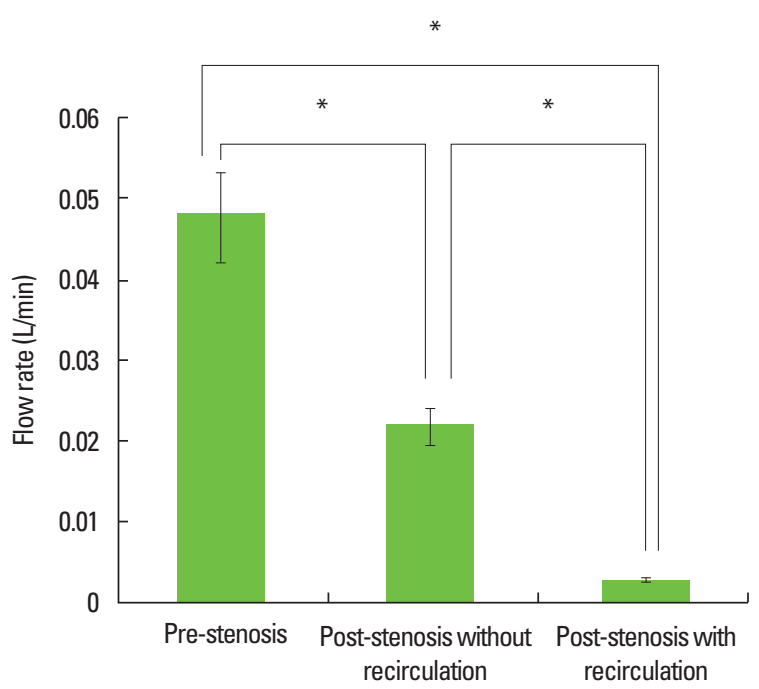

C

Figure 3. Hemodynamic estimation showing recirculating flow and decreased flow rate through perforator. (A) Recirculating flow in the post-stenotic area, $(\mathrm{B}$ and $\mathrm{C})$ Lower flow rates through microtubules inserted into the area with recirculating flow; $\mathrm{H}$ and $\mathrm{V}$ indicates the slice numbers of horizontal and vertical planes.

Table 1. Flow rates through microtubules inserted at each area of the 3Dprinted stenosis model

\begin{tabular}{llcc}
\hline & Pre-stenosis & $\begin{array}{c}\text { Post-stenosis without } \\
\text { recirculating flow }\end{array}$ & $\begin{array}{c}\text { Post-stenosis with } \\
\text { recirculating flow }\end{array}$ \\
\hline Experiment 1 & 56.00 & 18.75 & 2.61 \\
Experiment 2 & 44.21 & 20.87 & 2.73 \\
Experiment 3 & 46.66 & 22.86 & 2.47 \\
Experiment 4 & 42.00 & 23.10 & 2.73 \\
Experiment 5 & 49.41 & 24.00 & 2.57 \\
Mean [SD] $^{*}$ & $47.66[0.54]$ & $21.91[0.21]$ & $2.60[0.01]^{\dagger}$ \\
\hline
\end{tabular}

Results are expressed by flow rate $(\mathrm{mL} / \mathrm{min})$.

${ }^{*}$ Mean represents the mean and SD of the 5 experiments; ${ }^{~} T$ The flow rate of post-stenotic area with recirculating flow were statistically significantly different with the other two location $(P<0.001)$.

quently, the relatively low pressure in the recirculation flow region reduces the pressure gradient between the $\mathrm{BA}$ and the perforator, finally reducing the flow rate through the perforator at the post-stenosis region. Furthermore, this region is prone to ischemia and platelet activation, which may lead to cerebral infarction. ${ }^{11}$ In the present case, TOF-MRA showed an area with signal intensity remaining lower in the immediate post-stenotic area, though the diameter of the BA lumen was restored. This area well correspond with the area of recirculating flow shown in the PIV experiment. As the signal intensity of TOF-MRA is highly dependent on blood flow, decreased signal intensity in this region may reflect hemodynamic alterations in post-stenotic areas. ${ }^{12}$

Finally, the flow rate to microtubules was decreased in the area with recirculating flow, which may explain why the PPI occurred at the level of decreased signal intensity on TOFMRA. The pathomechanism of perforator infarction is evolv- 
ing using advanced brain imaging techniques. ${ }^{13}$ Some studies focused on the small atherosclerotic plaques causing perforator infarctions which were observed from HR-MRI, but not by conventional MRIs. ${ }^{14}$ However, those highly advanced imaging techniques still cannot demonstrate the hemodynamics of intracranial artery directly. For example, though dolichoectasia of the BA has been thought to be associated with the occurrence of PPI by a hemodynamic mechanism (stagnation or reversal of blood flow), ${ }^{15,16}$ the exact association could not be directly shown.

Based on the dynamic similarity in fluid mechanics, two geometrically similar vessels of different sizes with the same Reynolds number has hemodynamically identical flow structures. This allows the use of 3D-printed intracranial artery model and PIV to investigate the hemodynamic characteristics of flow in patients with various intracranial vascular diseases using in-vitro phantoms with variable scales. Though the hemodynamics can be observed from duplex sonography in the extracranial arteries, such as the carotid artery, there was no method to directly visualize the hemodynamic property of intracranial artery. Therefore, previously PIV was used to evaluate the hemodynamic characteristics of flow inside cerebral aneurysms and to identify rupture-prone aneurysms. ${ }^{17,18}$ As flowthrough phantoms made with silicone compounds are slightly distensible more likely as the arteries in-vivo with elasticity, PIV may closely reflect the real blood flow inside intracranial arteries. In addition, 3D-printed intracranial artery models can be modified, such as by inserting microtubules, which represent the perforators originating from the intracranial arteries. This allows it available to directly measure the hemodynamic alterations in the intracranial artery affecting the blood flow through perforators.

However, there are noteworthy limitations that should be considered in this model, especially when applied to intracranial arteries. Intracranial arteries have many branches and perforators. First, the vasculature is simplified during 3D-modeling by removing the branches of the parental artery, and this may bias the real flow dynamics of the parental artery. Adjustment for the threshold extracting the vasculature must be needed. Second, co-registration of HR-MRI and source image of TOF-MRA may have strengthened our result by showing the exact location of paramedian perforator causing PPI on the 3D-model. However, the level of perforators were confirmed from the scout image on TOF-MRA (Figure 1B, line $\mathrm{C}$ ), and the location of recirculating flow, where the microtubule were inserted, well-corresponds with the area with low signal intensity on TOF-MRA, which is on the level of perforators.
PPI can be caused by recirculating flow in post-stenotic areas, even without a plaque obliterating the orifice of the perforators. Although our method has limitations in reproducing the exact blood flow inside cerebral arteries, due to plaque-induced differences in arterial elasticity or reflecting the pulsatile nature of the blood flow, PIV is a useful tool to verify the pathomechanism of perforator infarction due to hemodynamic alterations in intracranial stenotic disease.

\section{References}

1. Bassetti C, Bogousslavsky J, Barth A, Regli F. Isolated infarcts of the pons. Neurology 1996;46:165-175.

2. Klein IF, Lavallée PC, Schouman-Claeys E, Amarenco P. Highresolution MRI identifies basilar artery plaques in paramedian pontine infarct. Neurology 2005;64:551-552.

3. Mehdiratta M, Caplan LR, Kumar S. Basilar artery branch disease imaged by magnetic resonance imaging. Arch Neurol 2007; 64:1666.

4. Kim BJ, Kwon SU. Perforator infarction immediately distal to the stenosis of parental artery: is it hemodynamic? J Stroke Cerebrovasc Dis 2014;23:1991-1993.

5. Choi JY, Choi JH, Kim NK, Kim Y, Lee JK, Kim MK, et al. Analysis of errors in medical rapid prototyping models. Int J Oral Maxillofac Surg 2002;31:23-32.

6. Ha H, Hwang D, Choi WR, Baek J, Lee SJ. Fluid-dynamic optimal design of helical vascular graft for stenotic disturbed flow. PLoS One 2014;9:e111047.

7. Wootton DM, Ku DN. Fluid mechanics of vascular systems, diseases, and thrombosis. Annu Rev Biomed Eng 1999;1:299-329.

8. Zhao R, Marhefka JN, Shu F, Hund SJ, Kameneva MV, Antaki JF. Micro-flow visualization of red blood cell-enhanced platelet concentration at sudden expansion. Ann Biomed Eng 2008;36: 1130-1141.

9. Jeong J, Hussain F. On the identification of a vortex. J Fluid Mech 1995;285:69-94.

10. Dubief Y, Delcayre F. On coherent-vortex identification in turbulence. J Turbul 2000;1:011. http://jot.iop.org

11. Zhang JN, Bergeron AL, Yu Q Sun C, McIntire LV, López JA, et al. Platelet aggregation and activation under complex patterns of shear stress. Thromb Haemost 2002;88:817-821.

12. Leng X, Wong KS, Soo Y, Leung T, Zou X, Wang Y, et al. Magnetic resonance angiography signal intensity as a marker of hemodynamic impairment in intracranial arterial stenosis. PLoS One 2013;8:e80124.

13. Norrving B. Evolving concept of small vessel disease through advanced brain imaging. J Stroke 2015;17:94-100.

14. Kim BJ, Yoon Y, Lee DH, Kang DW, Kwon SU, Kim JS. The 
shape of middle cerebral artery and plaque location: high-resolution MRI finding. Int J Stroke 2015;10:856-860.

15. Kwon HM, Kim JH, Lim JS, Park JH, Lee SH, Lee YS. Basilar artery dolichoectasia is associated with paramedian pontine infarction. Cerebrovasc Dis 2009;27:114-118.

16. Schwartz A, Rautenberg W, Hennerici M. Dolichoectatic intracranial arteries: review of selected aspects. Cerebrovasc Dis 1993; 3:273-279.
17. Yagi T, Sato A, Shinke M, Takahashi S, Tobe Y, Takao H, et al. Experimental insights into flow impingement in cerebral aneurysm by stereoscopic particle image velocimetry: transition from a laminar regime. J R Soc Interface 2013;10:20121031.

18. Goubergrits L, Thamsen B, Berthe A, Poethke J, Kertzscher U, Affeld $\mathrm{K}$, et al. In vitro study of near-wall flow in a cerebral aneurysm model with and without coils. AJNR Am J Neuroradiol 2010;31:1521-1528. 